\title{
Unpredictability of Delivered Bubble Nasal Continuous Positive Airway Pressure: Role of Bias Flow Magnitude and Nares-Prong Air Leaks
}

\author{
DORON J. KAHN, SHERRY E. COURTNEY, ANDREW M. STEELE, AND ROBERT H. HABIB \\ Department of Neonatal-Perinatal Medicine [D.J.K., S.E.C., A.M.S.], Schneider Children's Hospital, NSLIJHS, New Hyde Park, New York \\ 11040; Department of Pediatrics [R.H.H.], Mercy Children's Hospital at St. Vincent Mercy Medical Center and the University of \\ Toledo-College of Medicine, Toledo, Ohio 43608
}

\begin{abstract}
Nasal continuous-positive-airway-pressure (NCPAP) is popular for infant respiratory support. We compared delivered to intended intra-prong, proximal-airway, and distal-airway pressures using ventilator (V-NCPAP) and bubble (B-NCPAP) devices. Measurements were repeated at five flows $(4,6,8,10$, and $12 \mathrm{~L} / \mathrm{min})$ and three $\mathrm{NCPAP}\left(4,6\right.$, and $\left.8 \mathrm{~cm} \mathrm{H}_{2} \mathrm{O}\right)$ under no, small, and large nares-prong interface leak conditions. With no-leak, delivered BNCPAP was systematically greater than intended levels at all pressure sites. The corresponding V-NCPAP flow-dependence was noneto-minimal. Prong and intra-airway B-NCPAP overshoots were also observed with small-leak, while only prong B-NCPAP showed a flow-dependent overshoot for large-leak. Leaks did not affect intraprong V-NCPAP but resulted in progressively lower than desired, flow-independent intra-airway V-NCPAP. We conclude that the selfadjusting capability of ventilators allows closely matched actual versus intended V-NCPAP. Alternatively, for the range of flows used clinically, intra-prong and intra-airway B-NCPAP are systematically higher at increasing flows than operator-intended levels, even when appreciable nares-prong leak is present. Additionally, the oscillations (noise) characterizing B-NCPAP are substantially attenuated between the proximal and distal airways; therefore, it is unlikely that B-NCPAP engenders ventilation or lung recruitment via this phenomenon. Tubing submersion depth for setting the level of BNCPAP is highly inaccurate, and operators should instead rely on intra-prong pressure measurement. (Pediatr Res 62: 343-347, 2007)
\end{abstract}

$\mathrm{N}$ CPAP is an increasingly popular mode of respiratory support in preterm infants with varying pulmonary disorders. The renewed interest in this form of ventilatory support aims at preventing collapse of the alveoli and terminal airways during expiration while relying on the infant's spontaneous respiration, and thereby avoids both the risk of tracheal intubation and concerns about ventilator-induced lung injury $(1,2)$.

A variety of devices are currently used for NCPAP delivery in infants. Most commonly, an infant ventilator is used to generate positive pressure. Flow is continuous and NCPAP

Received December 12, 2006; accepted April 19, 2007

Correspondence: Sherry E. Courtney, M.D., Division of Neonatology, Room 344, Schneider Children's Hospital, 269-01 76 ${ }^{\text {th }}$ Avenue, New Hyde Park, NY 11040; e-mail: scourtne@lij.edu

Presented in part at the Society for Pediatric Research annual meeting, San Francisco, CA, May 2006, and the American Thoracic Society annual meeting, San Diego, CA, May 2006.

This study was supported in part by a grant from INO Therapeutics. pressure can be changed as desired by using the ventilator's positive end-expiratory pressure control. Ventilators maintain the delivered NCPAP pressure (V-NCPAP) close to the set pressure by automatic adjustments at the expiratory valve. Another device, B-NCPAP, has become a focus of interest as it is relatively inexpensive, easy to use, and favored by an institution reporting a low incidence of chronic lung disease (3). Warmed, humidified gas flows to the infant via the NCPAP circuit, binasal prongs are used, and the expiratory limb of the NCPAP tubing is submerged underwater to a depth in centimeters equal to the desired NCPAP. Flow rate is adjusted until gas bubbles through the water chamber. A hypothesized benefit to gas exchange and lung recruitment during B-NCPAP, the high-frequency oscillatory content of the bubbling, has been reported by some authors $(4,5)$ but not substantiated by others (6).

Most clinicians using B-NCPAP assume that the delivered mean intra-prong (and hence intra-airway) NCPAP in $\mathrm{cm} \mathrm{H}_{2} \mathrm{O}$ is accurately represented by the submersion depth of the expiratory tubing. However, B-NCPAP is characterized by its oscillatory (noisy) nature, caused by generation of the bubbles. How this characteristic affects the delivered mean BNCPAP is incompletely understood, especially since these inherent oscillations are themselves dependent on the bias flow rate. In addition, the importance of bubble oscillations on the effective intra-pulmonary B-NCPAP delivery is itself influenced by the extent of air leaks present at the nares-prong interface.

In a lung model, we compared the delivered intra-prong, proximal airway, and distal airway pressures during both B-NCPAP and V-NCPAP as a function of the desired (or set) NCPAP level and the bias flow. In addition, we characterized the impact of leaks at the nares-prong interface on B-NCPAP and V-NCPAP delivery.

\footnotetext{
Abbreviations: B-NCPAP, bubble nasal continuous positive airway pressure; NCPAP, nasal continuous positive airway pressure; Pdistal, pressure $\left(\mathrm{cm} \mathrm{H}_{2} \mathrm{O}\right)$ at the distal airway; Pprong, pressure $\left(\mathrm{cm} \mathrm{H}_{2} \mathrm{O}\right)$ at the nasal prongs; Pprox, pressure $\left(\mathrm{cm} \mathrm{H}_{2} \mathrm{O}\right)$ at the proximal airway; V-NCPAP, ventilatorgenerated nasal continuous positive airway pressure
} 


\section{METHODS}

Test lung model and instrumentation. A schematic of our experimental system is shown in Figure 1. We used a 1-L neonatal/pediatric test lung filled with copper wool (SensorMedics, Yorba Linda, CA). Calibrated compliance of the test lung was $1.03 \mathrm{~mL} / \mathrm{cm} \mathrm{H}_{2} \mathrm{O}$ and resistance was $0.06 \mathrm{~cm} \mathrm{H}_{2} \mathrm{O} / \mathrm{L} / \mathrm{min}$. To simulate nares-prong air leaks, Hudson NCPAP prongs (Hudson Respiratory Care Inc., Temecula, CA) size 1 were inserted into simulated nares fashioned from identical plastic caps with holes precisely cut to give "naresprong " area ratios of 1:1 (no leak), 1:1.25 (small leak), and 1:1.5 (large leak). The caps used can be viewed in the online supplement (Fig. S-1).

The neck of the test lung had an internal diameter of $0.5 \mathrm{~cm}$. Validyne DP45-28 pressure transducers (range $\pm 56 \mathrm{~cm} \mathrm{H}_{2} \mathrm{O}$, Validyne, Northridge, CA) were used to simultaneously measure Pprong, Pprox, and Pdistal. The pressure port of the Hudson prongs, located $3.5 \mathrm{~cm}$ from the midpoint of the prongs, was used for Pprong. The Pprox pressure transducer was located 5.5 $\mathrm{cm}$ distal to the simulated nares, and Pdistal was measured through a port at the bottom of the test lung. All transducers were simultaneously calibrated using a water manometer (Dwyer Instruments, Inc., Michigan City, IN). Data were collected using a computerized data acquisition system (Biopac Systems, Inc., Goleta, CA) at a sampling rate of 1000 samples per second per channel. V-NCPAP was given using a calibrated VIP Bird infant ventilator (Viasys Health Care, Inc., Yorba Linda, CA) with V-NCPAP provided using the positive end expiratory pressure control. For B-NCPAP, we submerged the expiratory limb of the ventilator tubing (length, $185 \mathrm{~cm}$; internal diameter, $1 \mathrm{~cm}$, noncorrugated tubing) in a chamber of water (diameter $7.5 \mathrm{~cm}$ ) to the level of NCPAP desired in $\mathrm{cm}_{2} \mathrm{O}$.

Measurements. Pprong, Pprox, and Pdistal data were collected at NCPAP settings of 4,6 , and $8 \mathrm{~cm} \mathrm{H}_{2} \mathrm{O}$ using both B-NCPAP and V-NCPAP as described. To determine the flow effects with each device at each NCPAP level, the gas flow was systematically increased in $2 \mathrm{~L} / \mathrm{min}$ increments from 4 to $12 \mathrm{~L} / \mathrm{min}$. Data were collected for $40-60 \mathrm{~s}$ at each flow rate. These same measurements were done using the three nares models simulating no-leak, small-leak, and large-leak conditions. In all cases, the effective delivered NCPAP at each point was estimated as the moving time-averaged pressure signal over 5-s windows. The lung model measurements were repeated and were found to be highly reproducible.

Role of B-NCPAP components. To confirm that our findings were not specific to the bubble system we used, we repeated the assessments following changes in its components. Specifically, using two sizes of nasal prongs (\#0 and \#1), we tested several variations of expiratory tubing and chamber size to assess how they might affect the flow-dependence during B-NCPAP: corrugated tubing, smaller and larger expiratory tubing diameter $(0.4$ and $2.0 \mathrm{~cm})$, shorter tubing length $(75 \mathrm{~cm})$, and a larger bubble chamber $(12.0 \mathrm{~cm}$ diameter).

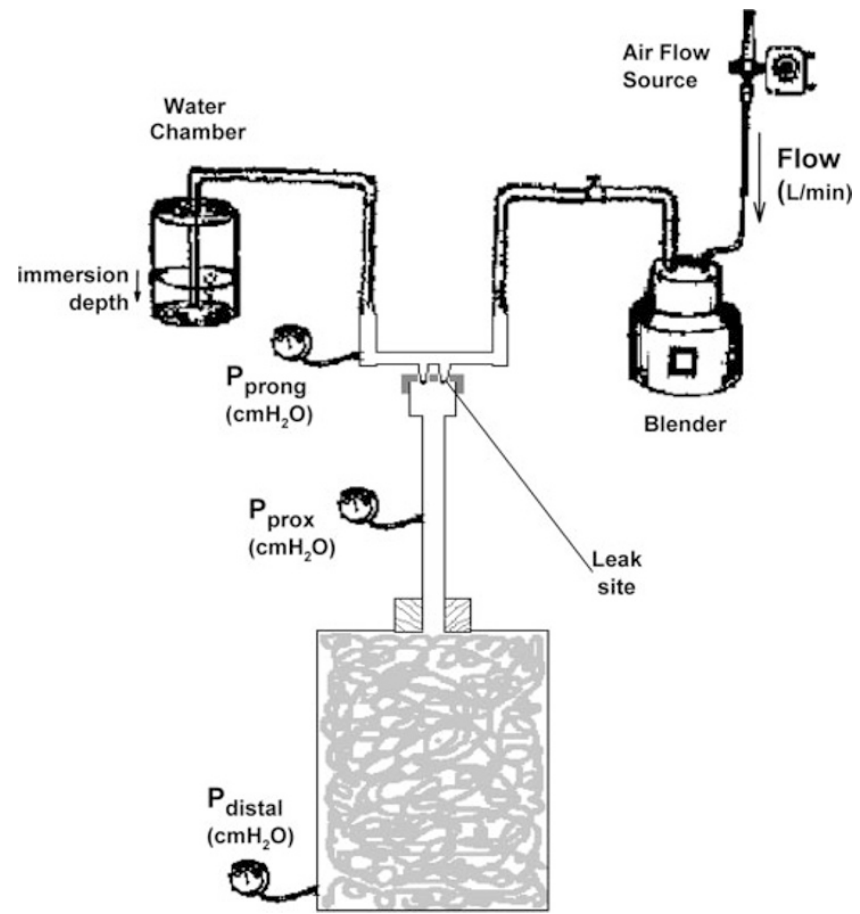

Figure 1. Schematic of the experimental test lung and B-NCPAP set-up.

\section{RESULTS}

Figure 2 shows example V-NCPAP and B-NCPAP pressure data obtained without leak at increasing flow levels. First, the delivered mean V-NCPAP closely approximated the desired value showing little variability with increasing flow levels at all three measurement sites. For B-NCPAP, the Pprong, Pprox, and Pdistal data exhibited large and systematic increases (larger at higher flow rates) in the mean delivered pressures relative to the desired NCPAP pressure set by submersion depth of the expiratory tubing. Also, these bubble pressures were characterized by their noisy (oscillatory) nature, with evidence of progressive attenuation of the oscillation amplitudes from the intra-prong pressure to the distal airway.

Figure 3 compares, at all flow rates, the effective B-NCPAP within the prongs to pressures in the proximal and distal airway, both with and without a leak at the nares-prong interface. The mean Pprox and Pdistal were essentially identical at all flow values and irrespective of expiratory tubing immersion depth or presence of leaks. Alternatively, Pprong mean pressures were always greater than Pprox and Pdistal with: 1) the difference increasing as flow increased; 2) the relative (or percentage) differences being more when the desired or set B-NCPAP was lower (more for 4 versus $8 \mathrm{~cm}$ immersion); and 3) greater differences in the presence of leak. Note, the increasing difference between Pprong and Pprox as flows were increased suggests a nonlinear effect likely secondary to increasing turbulence at higher flows.
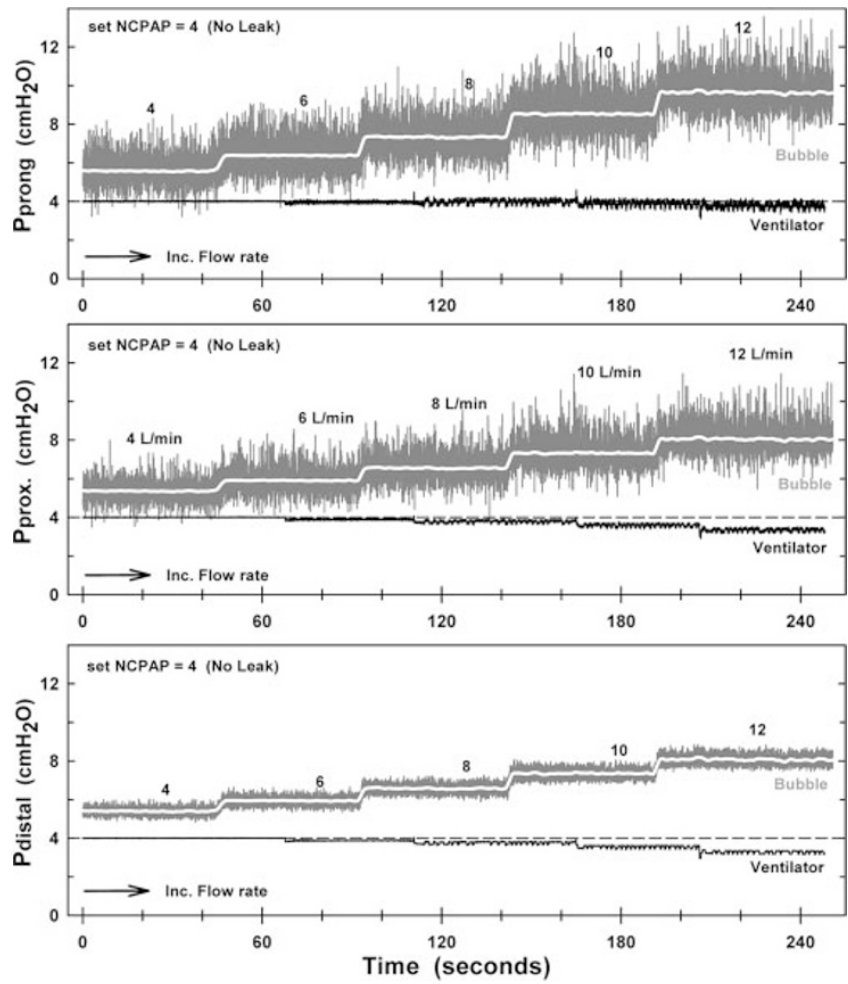

Figure 2. Effects of increasing flow on delivered NCPAP. Pprong, Pprox, and Pdistal tracings during B-NCPAP and V-NCPAP delivery at increasing flow rates between 4 and $12 \mathrm{~L} / \mathrm{min}$. Measurements shown reflect set NCPAP = $4 \mathrm{~cm} \mathrm{H}_{2} \mathrm{O}$ under no leak conditions. 

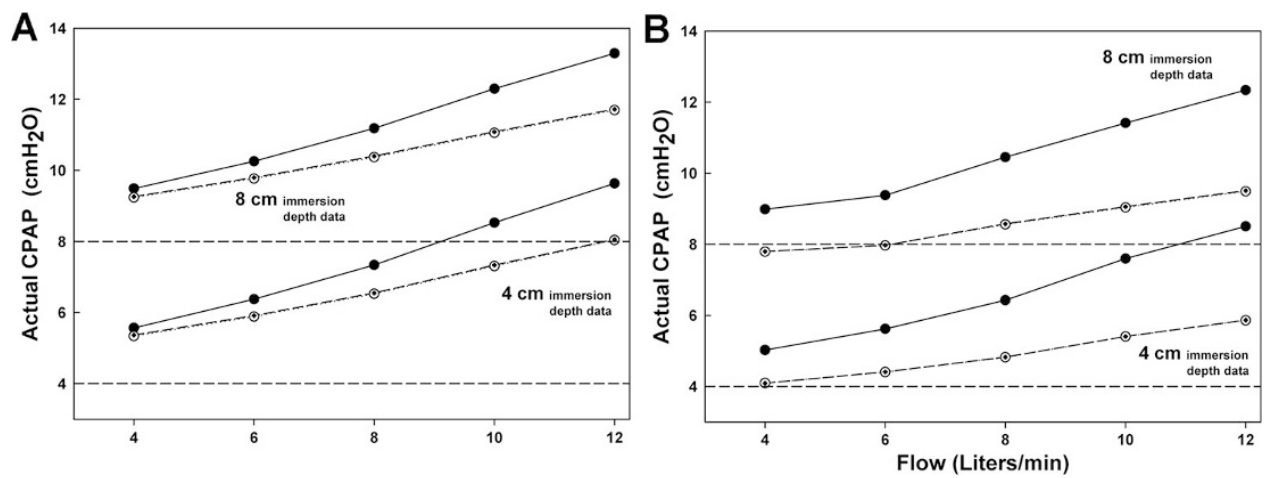

Figure 3. Actual B-NCPAP under no leak $(A)$ and small leak $(B)$ conditions shown for 4 and $8 \mathrm{~cm}$ expiratory tubing immersion depth in water. Pprong $(\bullet)$; Pprox $(\circ)$; Pdistal (small dot). Note that Pprox and Pdistal overlap.
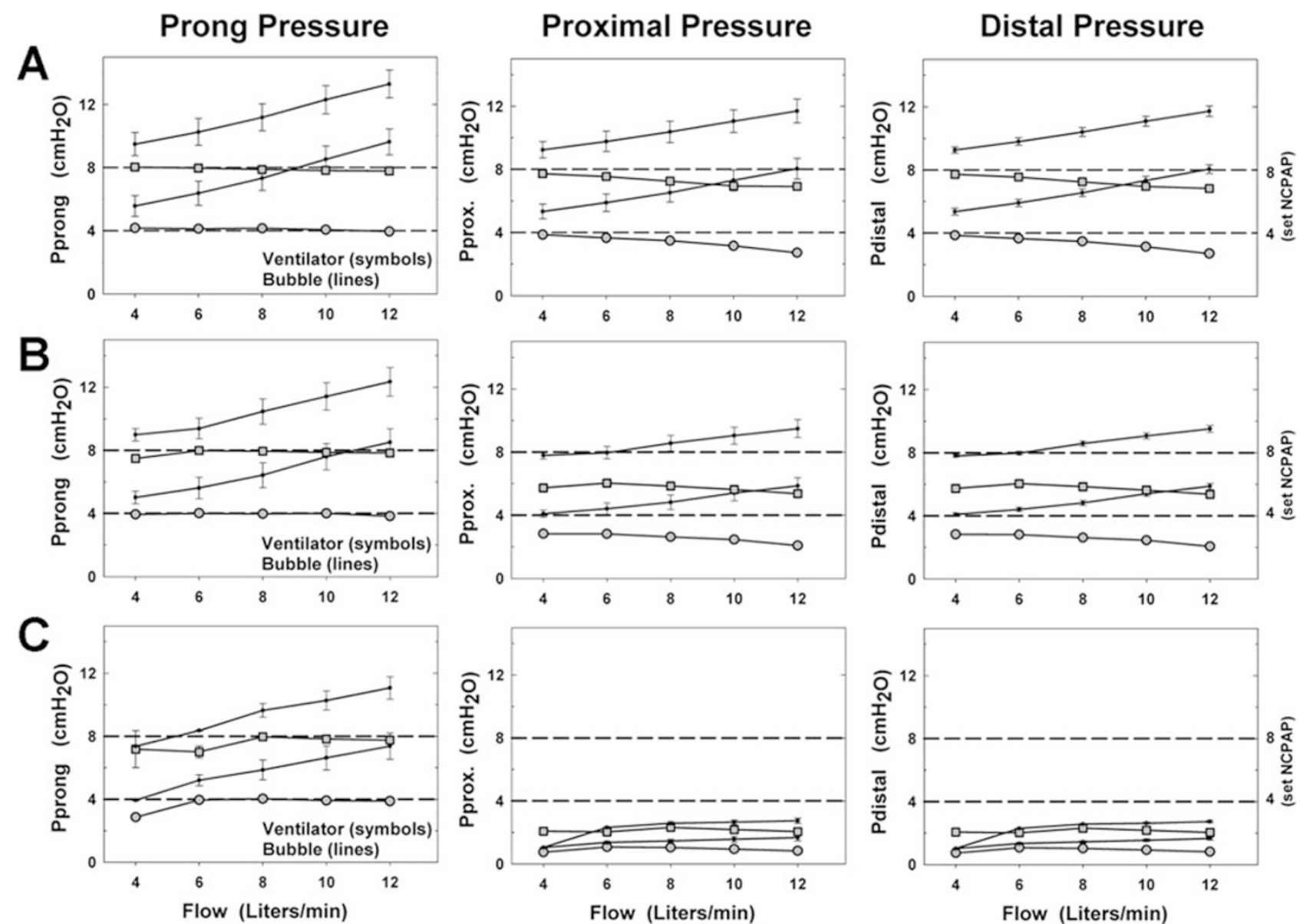

Figure 4. Comparison of delivered prong, proximal airway, and distal airway pressures during B-NCPAP and V-NCPAP for desired CPAP levels of 4 and 8 $\mathrm{cm} \mathrm{H}_{2} \mathrm{O}$. Data are repeated for no leak $(A)$, small leak $(B)$, and large leak $(C)$ conditions. The error bars seen for some of the B-NCPAP data points represent the SD derived from about 40,000 data points ( $40 \mathrm{~s}$ at $1000 \mathrm{~Hz}$ sampling rate; see Fig. 2 for no leak example). These error bars reflect the noise (oscillations) in the B-NCPAP pressure data (wider $=$ more noisy).

Figure 4 summarizes the overall experimental model results comparing V-NCPAP and B-NCPAP, and demonstrates the leak effects (none, small, and large) at all three pressure sites as a function of both flow and desired NCPAP (4 or $8 \mathrm{~cm}$ $\mathrm{H}_{2} \mathrm{O}$ ). The corresponding mean data-including the same data at set NCPAP of $6 \mathrm{~cm} \mathrm{H}_{2} \mathrm{O}$ - are provided in the online supplement (Table S-1). Briefly, as described above, delivered B-NCPAP at all three measurement sites were always increased at higher flows, and this flow effect was modulated by the extent of the leak present. In contrast, V-NCPAP pressures were generally flow-independent in the case of Pprong, and minimally flow-dependent (decreased) at high flows. The mean V-NCPAP at the Pprox and Pdistal site were identical with or without leak. Expectedly, when nares-prong leaks are present, Pprox and Pdistal will be substantially reduced relative to Pprong.

Lastly, irrespective of the NCPAP device used, our data demonstrate the ineffectiveness of intrapulmonary CPAP delivery when leaks are relatively large, such as for the 1:1.5 nares-prong area ratio.

Table 1 shows the effects of varying the expiratory tubing type, diameter, and length; as well as chamber size, at a tubing 
Table 1. Effects of B-NCPAP device component variations on intra-prong pressures (Pprong)* at $6 \mathrm{~cm} \mathrm{H}_{2} \mathrm{O}$ immersion depth

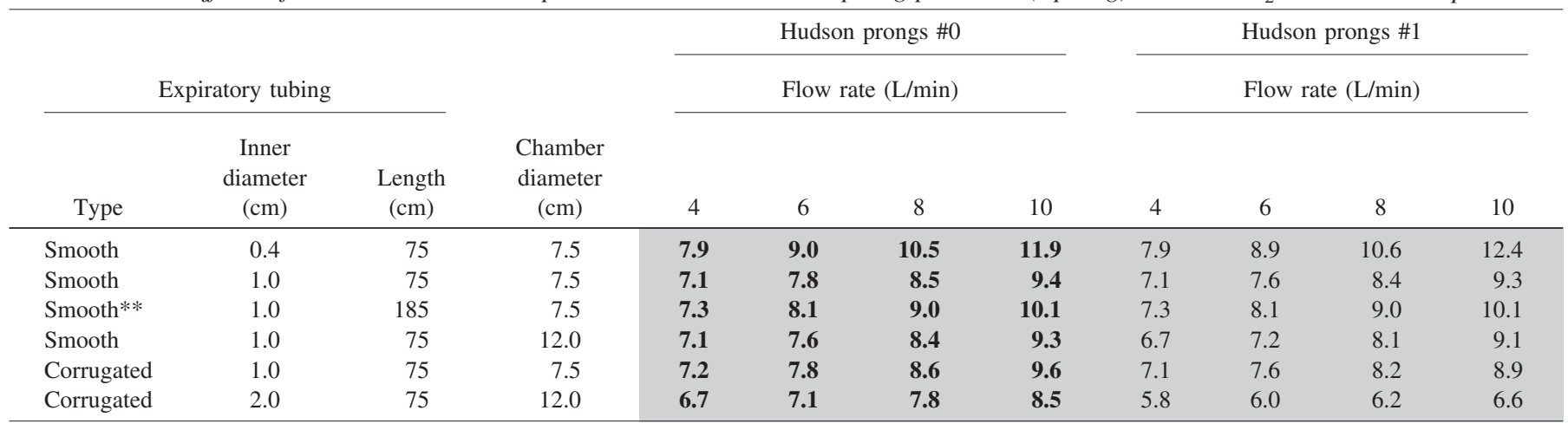

*The expected Pprong value (or B-NCPAP) in $\mathrm{cm} \mathrm{H}_{2} \mathrm{O}$ is equal to the expiratory tubing submersion depth, set at $6 \mathrm{~cm}$ in all cases under no-leak conditions. Data in shaded area reflect the actual or measured Pprong $\left(\mathrm{cm} \mathrm{H}_{2} \mathrm{O}\right)$ recorded at varying flow rates.

**System used in this study. Longer tubing length reflects that needed to switch between B-NCPAP and V-NCPAP without changing required setup.

immersion depth of $6 \mathrm{~cm} \mathrm{H}_{2} \mathrm{O}$. Tests were performed with both size \#0 (small) and size \#1 (larger) nasal prongs. All combinations tested demonstrated flow-dependence. More effect is seen with smaller tubing; the least effect is seen with the combination of the largest tubing, largest chamber, and largest nasal prongs.

\section{DISCUSSION}

Following demonstration of the efficacy of CPAP for treatment of infants with respiratory distress syndrome $(7,8)$ various modalities for CPAP delivery have been used. Although B-NCPAP has achieved considerable recent notoriety, few studies have examined whether it offers any treatment advantage over other forms of NCPAP delivery.

B-NCPAP is characterized by wide, noisy variations in peak-to-peak intra-prong pressures. Lee et al. (4) measured the frequency $(15-30 \mathrm{~Hz})$ and amplitude $\left(2-4 \mathrm{~cm} \mathrm{H}_{2} \mathrm{O}\right)$ of B-NCPAP oscillations in endotracheally intubated preterm infants (mean gestational age, $30.7 \mathrm{wk}$ ) ready for extubation and suggested that improvements in gas exchange might be due to delivery of low-amplitude, high-frequency oscillations to the lungs, somewhat analogous to the facilitated diffusion observed with high-frequency oscillatory ventilation. Pillow et al. (5) used a lung model to evaluate the effect of varying bias flow $(6-10 \mathrm{~L} / \mathrm{min})$ and lung compliance $(0.1-1.5 \mathrm{~mL} / \mathrm{cm}$ $\mathrm{H}_{2} \mathrm{O}$ ) on airway opening pressure fluctuations. They theorized that increasing magnitude and frequency of the "noisy" (no one single frequency) pressure wave form of B-NCPAP superimposed on pressure fluctuations as a result of spontaneous breathing may promote airway opening as a result of stochastic resonance (addition of "noise" to enhance output), perhaps reducing intrinsic work of breathing. They found that increasing bias flow and decreasing compliance, in their lung model, increased mean, magnitude, and frequency of pressure oscillations at the airway opening. They hypothesize from this that B-NCPAP may promote lung volume recruitment via stochastic resonance, augmenting the efficiency of gas mixing, especially in the poorly compliant lung.

Morley et al. (6) found a small but significant difference in NCPAP prong pressure when comparing slow bubbling (gas flow lowered to the point at which bubbling "just" occurred) to vigorous, high-amplitude bubbling (increasing gas flow above this level by $3 \mathrm{~L} / \mathrm{min}$ ). In this randomized crossover study of 26 preterm babies on B-NCPAP, these investigators found no difference in transcutaneous $\mathrm{CO}_{2}$, oxygen saturation, heart rate or respiratory rate, and suggested that pressure oscillations during B-NCPAP do not therefore improve gas exchange. Liptsen et al. (9) reported that the inspiratory work of breathing per milliliter of tidal volume delivered to the lungs was increased with B-NCPAP when compared with a variable-flow NCPAP. Blackson et al. (Blackson T, Pediatric Academic Societies' Annual Meeting, May 3-6, 2003, Seattle, WA) used a hot-wire anemometer attached to an endotracheal tube to measure oscillatory effect during B-CPAP in both a test lung and a neonatal piglet model. The found no significant ventilatory augmentation at gas flow rates of 7-10 $\mathrm{L} / \mathrm{min}$ either in vitro or in vivo.

In our study, we found the delivered V-NCPAP to be largely flow independent and B-NCPAP to be highly flow dependent, especially when leak is minimized. Leaks at the nares-prong interface modify both V-NCPAP and B-NCPAP, and the presence of large leaks compromises effective intraairway NCPAP delivery irrespective of the system used (see Pprox and Pdistal in Fig. 4). Intra-prong pressures were unaffected by leaks in the case of V-NCPAP, while for B-NCPAP these pressures were generally lower for all flows. These contrasting device data clearly reflected the lack of self-adjusting capabilities in the simple B-NCPAP system versus the compensation built into ventilator NCPAP delivery. These model results have important clinical implications since they indicate that delivered B-NCPAP exhibits substantial overshoot that is increased systematically as a function of the flow used. In relative terms, the percentage overshoot is greater when the clinically desired CPAP settings are lower (e.g. 4-5 $\mathrm{cm} \mathrm{H}_{2} \mathrm{O}$ compared with 7-8 $\mathrm{cm} \mathrm{H}_{2} \mathrm{O}$ ). This overshoot will also be greater as the leak is reduced. Indeed, the unpredictable nature of the leak adds to potential risks of providing dangerously high CPAP, such as if a sizeable leak, compensated for by increasing flow levels, is suddenly (and inadvertently) reduced or removed altogether.

Our model demonstrates when lung resistive properties (modeled by the copper wool in our test lung) are included, 
bubble oscillations are progressively and substantially attenuated distal to the level of the prongs (Fig. 2 and 4). These oscillations may be completely absent at the level of the distal airway when air leaks at the nares are present. Therefore, it is uncertain if and how B-NCPAP oscillations in the infant setting (where leaks are likely) will contribute to gas exchange (facilitated diffusion) or improved volume recruitment due to a hypothesized stochastic resonance. Even in no leak conditions, the substantial flow-dependence of actual versus desired (set) B-NCPAP raises the question of whether any improved recruitment is simply due to greater-than-desired B-NCPAP rather than the suggested stochastic resonance phenomenon. This issue is of particular importance when comparing BNCPAP to other forms of NCPAP, such as V-NCPAP or variable-flow NCPAP. We contend that studies contrasting the effectiveness of various NCPAP modalities should rigidly control the intra-prong pressures and standardize any allowed leaks to assure accurate comparisons between devices. Moreover, the data suggest that prong size should be chosen such that this ratio is less than 1:1.25 (small leak).

Total leak in infants on NCPAP will depend on prong and nares size, configuration of the nasal passages, and leak at the mouth. Chilton and Brooks (10) first described a reduction from set pressure to delivered pressure of approximately $50 \%$ when NCPAP pressures were sampled in the pharyngeal area of infants with open mouths, suggesting significant leak. De Paoli et al. (11) described 11 preterm infants receiving B-NCPAP whose mean pressure drop from prongs to pharynx was $2.2 \mathrm{~cm} \mathrm{H}_{2} \mathrm{O}$ with mouths closed and $3.2 \mathrm{~cm} \mathrm{H}_{2} \mathrm{O}$ with mouths open. Because B-NCPAP does not have built-in mechanisms that stabilize intra-prong pressures at varying flow rates, clinicians must carefully adjust the underwater tube immersion depth and flow rate combination, then confirm the delivered B-NCPAP via accurate intra-prong pressure monitoring.

A limitation of our study is that our findings may not be widely applicable due to differences in B-NCPAP device components among nurseries. We could not test every possible iteration of tubing and chamber differences. Yet, as we show in Table 1, substantial flow dependence is observed with B-NCPAP component variations that might be reasonably used in an intensive care nursery. The more limited (though still present) flow variation seen with $2.0-\mathrm{cm}$ expiratory tubing used with a large $(12-\mathrm{cm})$ diameter chamber and large (\#1) nasal prongs confirms the findings of Christensen et al. (12). They used a bubble device as an example of an almost ideal flow-independent threshold resistor, and found that positive end-expiratory pressure generated by an underwater seal with expiratory tubing width of $22 \mathrm{~mm}$ in a large chamber $(12 \times$ $18 \times 24 \mathrm{~cm}$ ) exhibited only a "minor degree" of flowdependence across a wide range of flows $(0-60 \mathrm{~L} / \mathrm{min})$. However, we have shown that what they determined to be a minor degree of flow dependence under these conditions becomes much greater with the B-NCPAP used in preterm infants.

It is also important to note that our bench study results reflect controlled in vitro conditions that may not necessarily correlate with NCPAP delivery in infants. The latter is far more complex and may be substantially influenced by other factors such as the underlying lung and chest wall mechanical properties. To more accurately determine the true distending pressures in infants on NCPAP, it is crucial that esophageal pressures (as estimates of intrapleural pressures) and/or pharyngeal pressures be measured.

Summary. Our data demonstrate that delivered B-NCPAP may vary considerably from the pressure set by the operator, especially when high flows are used. We also demonstrate the need to minimize leaks for consistent NCPAP delivery, irrespective of device. Because bubble systems do not have the built-in compensatory mechanisms necessary to stabilize delivered pressures, leak effects on NCPAP delivery are greater than observed with V-NCPAP, and these effects are themselves flow-dependent. When the test-lung model includes a dissipation element (representing airway resistance), we report a dramatic attenuation of pressure oscillation amplitudes in the distal airway suggesting that the oscillations (noise) caused by bubbling play a minimal, if any, role in ventilation or lung recruitment. Lastly, B-NCPAP users should be aware that tubing submersion depth is a highly inaccurate and unreliable estimate of actual delivered B-NCPAP and, thus, operators should instead rely on direct intra-prong pressure measurements. Unfortunately, even doing so, the operator will not completely remove the unpredictability of B-NCPAP delivery since it is highly sensitive to changes of the leak magnitude at the nares-prong interface.

Acknowledgments. The authors thank Michael Weisner, MSBE, for his technical assistance.

\section{REFERENCES}

1. Clark RH, Gerstmann DR, Jobe AH, Moffitt ST, Slutsky AS, Yoder BA 2001 Lung injury in neonates: causes, strategies for prevention, and long-term consequences. J Pediatr 139:478-486

2. Carney D, DiRocco J, Nieman G 2005 Dynamic alveolar mechanics and ventilatorinduced lung injury. Crit Care Med 33:S122-S128

3. Avery ME, Tooley WH, Keller JB, Hurd SS, Bryan MH, Cotton RB 1987 Is chronic lung disease in low birth weight infants preventable? A survey of eight centers. Pediatrics 79:26-30

4. Lee KS, Dunn MS, Fenwick M, Shennan AT 1998 A comparison of underwater bubble continuous positive airway pressure with ventilator-derived continuous positive airway pressure in premature neonates ready for extubation. Biol Neonate 73:69-75

5. Pillow JJ, Travadi JN 2005 Bubble CPAP: is noise important? An in vitro study. Pediatr Res 57:826-830

6. Morley CJ, Lau R, De Paoli A, Davis PG 2005 Nasal continuous positive airway pressure: does bubbling improve gas exchange? Arch Dis Child Fetal Neonatal Ed 90:F343-F344

7. Gregory GA, Kitterman JA, Phibbs RH, Tooley WH, Hamilton WK 1971 Treatment of the idiopathic respiratory distress syndrome with continuous positive airway pressure. N Engl J Med 284:1333-1340

8. Kattwinkel J, Fleming D, Cha CC, Fanaroff AA, Klaus MH 1973 A device for administration of continuous positive airway pressure by the nasal route. Pediatrics 52:131-134

9. Liptsen E, Aghai ZH, Pyon KH, Saslow JG, Nakhla T, Long J, Steele AM, Habib RH, Courtney SE 2005 Work of breathing during nasal continuous positive airway pressure in preterm infants: a comparison of bubble versus variable-flow devices. J Perinatol 25:453-458

10. Chilton HW, Brooks JG 1979 Pharyngeal pressures in nasal CPAP. J Pediatr 94:808-810

11. De Paoli AG, Lau R, Davis PG, Morley CJ 2005 Pharyngeal pressure in preterm infants receiving nasal continuous positive airway pressure. Arch Dis Child Fetal Neonatal Ed 90:F79-F81

12. Christensen EF, Jensen RH, Schonemann NK, Pedersen KD 1995 Flow-dependent properties of positive expiratory pressure devices. Monaldi Arch Chest Dis 50:150 153 